\title{
Autoevaluación y co-evaluación continua del trabajo individual y grupal en una asignatura experimental.
}

\section{Amparo Bes-Piáa, Beatriz Cuartas-Uribe ${ }^{b}$, Alicia Iborra-Clar ${ }^{c}$, M. Isabel Iborra- Clar $^{d}$ y Esperanza M. Garcia-Castello}

${ }^{a}$ Universitat Politècnica de València, mbespia@iqn.upv.es,${ }^{b}$ Universitat Politècnica de València, beacuau1@iqn.upv.es, ${ }^{c}$ Universitat Politècnica de València, aiborra@iqn.upv.es, ${ }^{d}$ Universitat Politècnica de València, miborra@iqn.upv.es é Universitat Politècnica de València, egarcia1@iqn.upv.es.

\begin{abstract}
This work is based on the evaluation of fundamental competence "teamwork" in order to get an assessment that reflects the work of each student within their group in an experimental subject. For this, the student participates in both selfassessment and co-assessment. Each evaluation process includes qualitative assessment of the group performance and quantitative assessment of academic work according to their degree of involvement.

The performance evaluation group is done through anonymous surveys based on the models of A. Garcia-Carbonell and F. Watts. In them, each student carries out an evaluation of each of its members and himself in two different moments of the semester: initial and final. In the quantitative assessment, students distribute the mark of the academic work according to the evaluation criteria of P. Morales-Vallejo.

Simultaneously teachers make an quantitative assessment of the academic work and the effectiveness of teamwork by means an assessment rubric.

The aim of these assessment methodologies is to identify students that do not contribute to the effectiveness of the group. So the marks for the academic works will depend on the degree of participation of each student to get a fairer individual assessment in an entirely experimental subject, where the teamwork is essential.
\end{abstract}

Keywords: experimental subject, teamwork competence, co-evaluation, selfevaluation. 


\section{Resumen}

Este trabajo se basa en la evaluación de la competencia básica "trabajo en grupo" con el fin de obtener una evaluación que refleje el trabajo de cada alumno dentro de su grupo en una asignatura experimental. Para ello, el alumno participa en un proceso de evaluación tanto individual (autoevaluación) como del resto de compañeros del grupo (co-evaluación). Cada evaluación contempla la valoración cualitativa del funcionamiento de los miembros del grupo y la valoración cuantitativa del trabajo académico realizado en función del grado de participación.

La evaluación del funcionamiento del grupo se realiza a través de encuestas anónimas basándose en los modelos de A. García-Carbonell y F. Watts. En ellas, cada alumno realiza una evaluación de cada uno de sus miembros y de él mismo en dos momentos diferentes del semestre: inicial y final. En la valoración cuantitativa, los alumnos distribuyen la nota obtenida en el trabajo académico según los criterios de P. Morales-Vallejo.

Paralelamente los profesores realizan la valoración de los trabajos académicos realizados y la efectividad del trabajo en grupo a través de 'rúbricas de evaluación'.

Con estas metodologías de evaluación se pretende detectar a los alumnos que no contribuyen a la efectividad del grupo, de forma que la nota de los trabajos académicos dependa del grado de participación de cada alumno. En definitiva, obtener una evaluación individual más justa en una asignatura completamente experimental, donde el trabajo en grupo es clave.

Palabras clave: asignatura experimental, trabajo en grupo, co-evaluación, autoevaluación. 
Bes-Piá, M.A., Cuartas-Uribe B., Iborra-Clar A., Iborra-Clar M.I., García-Castello E.

\section{Introducción}

En los nuevos planes de estudios, las titulaciones están basadas en la obtención de competencias transversales y específicas para cada titulación. Como consecuencia, una formación universitaria por competencias debe diseñar acciones que promuevan en los alumnos el aprendizaje activo y significativo a nivel conceptual, procedimental y actitudinal, donde la labor docente tenga un rol de facilitador del aprendizaje.

Una competencia transversal fundamental es el "trabajo en grupo". Esta competencia de tipo interpersonal, y por tanto, social y emocional, es necesaria a lo largo de la vida personal y profesional. El sociólogo Elton Mayo (1927), ya indicaba en sus estudios que "la participación y las relaciones interpersonales aumentan la productividad, la calidad de productos y salud de trabajadores“. Lograr un buen grupo de trabajo requiere de coordinación y organización con los demás, capacidad de adaptación, capacidad de escucha, autocrítica y compromiso de aprendizaje tanto propio como de los demás. Todo esto puede conseguirse a través del "aprender haciendo" que lleva implícito el trabajo en grupo.

Sin embargo, realizar una evaluación justa de esta competencia no es tarea fácil en general y, más aún, cuando parte del trabajo debe realizarse fuera del aula o laboratorio. Por lo tanto, la evaluación de un trabajo académico, realizado en estas circunstancias, no debe ser evaluado únicamente por el profesor y debe contar con la participación del alumno. Para ello, es preciso contar con metodologías que ayuden a recopilar y evidenciar el grado de adquisición de esta competencia.

Consecuentemente, este trabajo ahonda en la idea de evaluar la competencia "trabajo en grupo" en una asignatura completamente experimental. El trabajo presentado en InRed 2014 se amplía, a partir de la realización de una experiencia piloto que combina diferentes metodologías de innovación, con la finalidad de evaluar el funcionamiento de grupos de trabajo, tanto dentro como fuera del aula, y conseguir evaluaciones más justas.

\section{Objetivos}

- Detectar el grado de participación de los alumnos en su grupo de trabajo.

- Fomentar la actitud de auto-crítica en los alumnos.

- Obtener evaluaciones individuales más justas dentro del trabajo en grupo de una asignatura experimental.

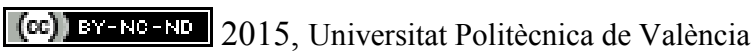

Congreso In-Red (2015) 


\section{Desarrollo de la innovación}

La innovación docente se ha llevado a cabo en la asignatura "Experimentación en Ingeniería Química I", de la titulación de grado de Ingeniero Químico, la cual consta únicamente de prácticas de laboratorio. Los alumnos matriculados (65 en el curso 20142015) se distribuyen libremente en grupos de 3 ó 4 componentes. En cada grupo, los alumnos realizan las prácticas de laboratorio y el "trabajo académico" (TA) asociado a cada práctica (memorias). Las prácticas de la asignatura se realizan todas en el laboratorio mientras que el trabajo académico no se realiza en las mismas instalaciones.

Las metodologías de evaluación utilizadas principalmente en este trabajo son: evaluación del funcionamiento del grupo y distribución de la nota del trabajo académico.

\subsection{Evaluación del funcionamiento del grupo}

La metodología empleada para la evaluación del funcionamiento de grupos de trabajo está basada en la denominada "Evaluación en tres tiempos" de A. García-Carbonell y F. Watts. En ella, cada alumno realiza de forma anónima una evaluación del funcionamiento del grupo en general, de cada uno de sus miembros (co-evaluación) y de él mismo (autoevaluación) a nivel cualitativo en los términos Insuficiente, Regular, Buena, Muy buena y Excelente. Además, esta evaluación se ha realizado en dos momentos diferentes del semestre: inicial y final. La evaluación se realiza cuando cada grupo entrega la memoria al profesor a través de un modelo de encuesta que se completa en muy poco tiempo.

Mediante esta metodología se evalúa la actitud de los miembros del grupo y no los conocimientos, ni procedimientos asociados a los contenidos de la asignatura.

\subsection{Distribución de la nota del trabajo académico}

En esta parte, el alumno realiza una evaluación cuantitativa del trabajo académico realizado en grupo siguiendo los criterios de evaluación de P. Morales-Vallejo. La propuesta de este autor consiste en multiplicar la nota obtenida en el trabajo académico (producto), que ha sido evaluado por el profesor, por el número de componentes del grupo (entre 3 y 4). El valor resultante es distribuido, por cada alumno, entre todos los componentes de su grupo, incluido el mismo, en función de cuál haya sido el grado de contribución de cada uno a la tarea común (evaluación del proceso).

Para esta parte se han seleccionado dos del total de los seis trabajos académicos realizados, uno al inicio (Trabajo académico 1) y otro al final del semestre (Trabajo académico 6). Dicha selección se corresponde con los tiempos de evaluación inicial y final del funcionamiento del grupo (apartado 3.1).

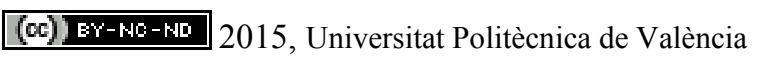


Bes-Piá, M.A., Cuartas-Uribe B., Iborra-Clar A., Iborra-Clar M.I., García-Castello E.

Finalmente, con la información obtenida en las metodologías descritas en los apartados 3.1 y 3.2 se busca ver qué relaciones existen entre la evaluación del funcionamiento del grupo con la distribución de la nota del trabajo académico. Adicionalmente, se comparará si la valoración realizada por los profesores a través de la 'rúbrica de evaluación' en términos de organización, coordinación tareas y participación activa se corresponde con la de los alumnos.

\section{Resultados}

\subsection{Evaluación del funcionamiento del grupo}

A continuación se muestran los resultados más representativos de la evaluación realizada por los alumnos sobre el funcionamiento de los grupos. En concreto, se han seleccionado tres grupos del total de los 18 evaluados, ya que pueden considerarse representativos del total de grupos evaluados.

Como se puede observar en las figuras 1, 2 y 3 cada uno de los grupos presentó un comportamiento diferenciado. En el grupo A (Fig. 1), todos los componentes del grupo tienen en general una opinión positiva del resto de compañeros y de ellos mismos. Cabe destacar una evaluación mayoritariamente Muy buena al inicio del curso. Además, en este grupo se observó una tendencia de evaluación creciente hacia Excelente, tal y como se aprecia en la evaluación final. La percepción que tienen los alumnos de este grupo coincide con la de los profesores, evaluada a través de la 'rúbrica de evaluación', pues es un grupo que se caracterizó por trabajar muy bien en el laboratorio y tener una actitud receptiva.

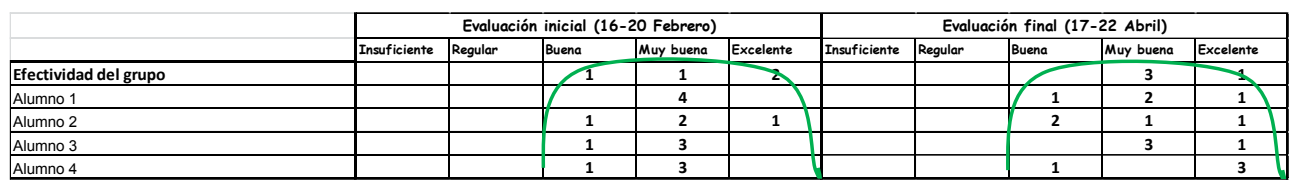

Fig. 1. Resultados funcionamiento del grupo A.

El grupo B (Fig. 2), se caracteriza inicialmente porque los componentes del grupo tienen también una opinión positiva del resto de compañeros y de ellos mismos. Sin embargo, a diferencia del grupo A, la opinión es en general prácticamente unánime tanto del grupo como del resto de compañeros, es lo que denominamos actitud 'corporativista' donde los compañeros no hacen apenas diferenciaciones entre compañeros. Esto podría indicar una falta de actitud crítica o también a que no han tenido suficiente tiempo para conocerse.

Sin embargo, con el tiempo se observa cómo la evaluación inicial cambia ampliándose la franja desde Insuficiente hasta Muy buena. En concreto, esto se atribuye a la poca

\section{(c)) EY-NC-ND 2015, Universitat Politècnica de València}


implicación de uno de los componentes del grupo. Este hecho también fue detectado por el profesorado a través de las 'rúbricas de evaluación'.

\begin{tabular}{|l|l|l|l|l|l|l|l|l|l|l|}
\cline { 2 - 10 } \multicolumn{1}{c|}{} & \multicolumn{3}{c|}{ Evaluación inicial (16-20 Febrero) } & \multicolumn{3}{c|}{ Evaluación final (17-22 Abril) } \\
\cline { 2 - 11 } & Insuficiente & Regular & Buena & Muy buena & Excelente & Insuficiente & Regular & Buena & Muy buena & Excelente \\
\hline Efectividad del grupo & & & & & & & & & & \\
\hline
\end{tabular}

Fig. 2. Resultados funcionamiento del grupo B.

En la Figura 3, se observa como inicialmente el grupo $\mathrm{C}$ tiene una opinión excelente tanto del funcionamiento del grupo como de los componentes del mismo. Además en este grupo, al igual que el grupo B, se podría hablar también de 'actitud corporativista'. Consideramos que en parte es normal si tenemos en cuenta que en la evaluación inicial los grupos sólo han realizado un trabajo académico.

Sin embargo, en la evaluación final, la opinión de los componentes se ha dispersado mucho, tal y como muestra la amplitud de la curva, y la calificación Excelente ya no se considera ni a nivel de grupo ni a nivel individual. La dispersión se debe a que uno de los componentes del grupo no trabaja como el resto desearía. Sin embargo, los profesores evaluaron positivamente la actitud y el trabajo en grupo realizado en el laboratorio a través de las 'rúbricas de evaluación' a todos los componentes sin excepción. Esto indica que trabajar en grupo en el laboratorio no implica hacerlo fuera del mismo y este aspecto debe ser tenido en cuenta en la nota del trabajo académico de cada alumno.

\begin{tabular}{|c|c|c|c|c|c|c|c|c|c|c|}
\hline & \multicolumn{5}{|c|}{ Evaluación inicial (16-20 Febrero) } & \multicolumn{5}{|c|}{ Evaluación final (17-22 Abril) } \\
\hline & Insuficiente & Regular & Buena & Muy buena & Excelente & Insuficiente & Regular & Buena & Muy buena & Excelente \\
\hline Efectividad del grupo & & & & 3 & & & 1 & 2 & 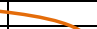 & \\
\hline Alumno 1 & & & 1 & 25 & & & 7 & 1 & 2 & \\
\hline Alumno 2 & & & & 3 & & 2 & & 1 & 2 & \\
\hline Alumno 3 & & & 1 & 3 & & 1 & & 1 & 1 & \\
\hline
\end{tabular}

Fig. 3. Resultados funcionamiento del grupo $C$.

\subsection{Distribución de la nota del trabajo académico}

En la Tabla 1 se recogen las notas de los trabajos académicos evaluados por los profesores (TA), las notas de los trabajos académicos a distribuir (AD), la distribución de la nota realizada por cada alumno y la media de las notas distribuidas por los alumnos (MN). 
Bes-Piá, M.A., Cuartas-Uribe B., Iborra-Clar A., Iborra-Clar M.I., García-Castello E.

Tabla 1. Evaluación de los trabajos académicos por parte de los profesores y alumnos

\begin{tabular}{|c|c|c|c|c|c|c|c|c|c|}
\hline GRUPO & NOTA & \multicolumn{4}{|c|}{$\begin{array}{l}\text { TRABAJO ACADÉMICO } 1 \\
\text { (INICIAL) }\end{array}$} & \multicolumn{4}{|c|}{$\begin{array}{l}\text { TRABAJO ACADÉMICO } 6 \\
\text { (FINAL) }\end{array}$} \\
\hline \multirow{8}{*}{ A } & Nota TA & \multicolumn{4}{|c|}{9,0} & \multicolumn{4}{|c|}{9,8} \\
\hline & Nota AD & \multicolumn{4}{|c|}{$9,0 \times 4=36$} & \multicolumn{4}{|c|}{$9,8 \times 4=39,2$} \\
\hline & Alumno & 1 & 2 & 3 & 4 & 1 & 2 & 3 & 4 \\
\hline & 1 & 8 & 8 & 10 & 10 & 9,6 & 9,6 & 10 & 10 \\
\hline & 2 & 9 & 8 & 9 & 10 & 9,8 & 9,6 & 9,8 & 10 \\
\hline & 3 & 10 & 10 & 8 & 8 & 9,8 & 9,8 & 9,8 & 9,8 \\
\hline & 4 & 8,5 & 8,5 & 10 & 9 & 9,73 & 9,73 & 10 & 9,74 \\
\hline & $\mathrm{MN}$ & 8,87 & 8,62 & 9,25 & 9,25 & 9,73 & 9,68 & 9,90 & 9,88 \\
\hline \multirow{6}{*}{ B } & Nota TA & \multicolumn{4}{|c|}{6,8} & \multicolumn{4}{|c|}{5,0} \\
\hline & Nota AD & \multicolumn{4}{|c|}{$6,8 \times 3=20,4$} & \multicolumn{4}{|c|}{$5,0 \times 3=15$} \\
\hline & 1 & 6,4 & 7 & 7 & & 4,5 & 6,0 & 4,5 & \\
\hline & 2 & 6,8 & 6,5 & 7,1 & & 5,5 & 5,5 & 4,0 & \\
\hline & 3 & 8,3 & 8,3 & 3,8 & & 5,5 & 5,5 & 4,0 & \\
\hline & $\mathrm{MN}$ & 7,16 & 7,26 & 5,96 & & 5,16 & 5,66 & 4,16 & \\
\hline \multirow{7}{*}{ C } & Nota TA & \multicolumn{4}{|c|}{5,4} & \multicolumn{4}{|c|}{3,5} \\
\hline & Nota AD & \multicolumn{4}{|c|}{$5,4 \times 3=16,2$} & \multicolumn{4}{|c|}{$3,5 \times 4=14$} \\
\hline & 1 & 5,4 & 5,4 & 5,4 & - & 3,5 & 3,5 & 3,5 & 3,5 \\
\hline & 2 & 5,4 & 5,4 & 5,4 & - & 3,5 & 3,5 & 3,5 & 3,5 \\
\hline & 3 & 5,4 & 5,4 & 5,4 & - & 3,5 & 3,5 & 3,5 & 3,5 \\
\hline & 4 & - & - & - & - & 3,5 & 3,5 & 3,5 & 3,5 \\
\hline & $\mathrm{MN}$ & 5,4 & 5,4 & 5,4 & - & 3,5 & 3,5 & 3,5 & 3,5 \\
\hline
\end{tabular}

TA: trabajo académico, AD: nota del TA a distribuir, NE: no evaluado, MN: media notas

Grupo A: este grupo se caracteriza por haber obtenido en los trabajos académicos (TA) calificaciones muy buenas durante el transcurso de la asignatura. Se observa que los alumnos tienen una actitud crítica a la hora de evaluar tanto los compañeros como a ellos mismos. En general, los alumnos se puntúan con nota inferior o igual que al resto de compañeros. Esto se puede observar en la distribución de la nota de los componentes 1 y 3 donde se invierten las puntuaciones, al considerar que la contribución al trabajo académico realizado por los compañeros es mejor que el propio.

En general, la media de las notas (MN) obtenidas por cada uno de los componentes se mueve en torno a la nota del TA, encontrándose pequeñas diferencias entre los miembros del grupo en la evaluación final. Esto significa que todos los miembros del grupo

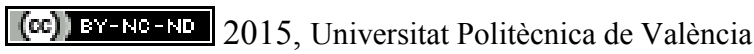

Congreso In-Red (2015) 
contribuyeron de forma equitativa al trabajo académico realizado en el grupo. Además, esta tendencia se mantuvo y se reforzó en el tiempo ya que las diferencias entre las notas del TA y MN se redujo. Estos resultados se pueden correlacionar con la evaluación del funcionamiento del grupo (Fig. 1), en la cual se refleja como un grupo que inicialmente trabaja bien en equipo con el tiempo refuerza más esta competencia.

Grupo B: este grupo se caracteriza por haber obtenido unas calificaciones medias en los trabajos académicos (TA) de la asignatura. Como en el grupo anterior, se sigue la tendencia en la que las notas de la co-evaluación son mejores que en la autoevaluación. En este sentido, cabe destacar que la autoevaluación de uno sus miembros es muy inferior a las de sus compañeros. En concreto, el componente tres de este grupo puntuó inicialmente su contribución en el 'trabajo académico 1' con una nota de 3,8 sobre el total 6,8 mientras que sus compañeros le puntuaron con notas en torno a 7/6,8 (Tabla 1). No obstante, la percepción de los componentes 1 y 2 sobre 3 cambió en la evaluación final, aumentado la diferencia entre la nota del 'trabajo académico 6' (TA) y la media de notas de la distribución (MN) con un valor de MN/TA=4,16/5,0.

Cabe resaltar además que, para este componente, se aproximan las notas obtenidas en la coevaluación $(4,0 / 5$ y $4,5 / 5)$ y la autoevaluación $(4,0 / 5)$. Sin embargo, este resultado no concuerda con la evaluación final del funcionamiento de este grupo (Fig.2), donde el alumno 3 evalúa su labor dentro del grupo como Insuficiente mientras que el resto de compañeros la evalúa como Buena. Esto podría deberse a que el alumno tiene una actitud muy crítica consigo mismo por la contribución realizada al trabajo de grupo, mientras que sus compañeros no perciben esto de igual forma.

Grupo C: este grupo se caracteriza por haber obtenido las peores calificaciones en los TA de la asignatura. Se observa que los alumnos no tienen una actitud crítica a la hora de distribuir las notas de ambos trabajos académicos y todos se puntúan de igual forma, tal y como se recoge en la Tabla 1.

Sin embargo, si se relacionan estos resultados con la evaluación del funcionamiento del grupo, cabe destacar que, a pesar de las notas de los TA, siguen considerando que trabajan muy bien en grupo, tanto dentro como fuera del aula, manteniéndose las evaluaciones en torno a Muy buena y Buena y con una ligera cola de la campana hacia Regular (Fig.3) provocada por uno de los componentes del grupo. Sin embargo, esto no se refleja en la distribución de las notas manteniéndose una actitud corporativista. A pesar de ello, los componentes de este grupo tienen una actitud muy receptiva hacia las recomendaciones de los profesores así como una evaluación positiva en las 'rúbricas de evaluación', como ya se comentó. 
Bes-Piá, M.A., Cuartas-Uribe B., Iborra-Clar A., Iborra-Clar M.I., García-Castello E.

\section{Conclusiones}

Mediante la evaluación del funcionamiento del grupo y la distribución de la nota del trabajo académico que realizan los alumnos es posible detectar aquellos alumnos que contribuyen en menor y en mayor medida a la realización del trabajo en común.

Las evaluaciones del profesorado a través de las 'rúbricas de evaluación' realizadas en el laboratorio discrepan en ocasiones de las co-evaluaciones y auto-evaluaciones debido a la limitación espacio-temporal.

Los actos de co-evaluación y auto-evaluación fomentan la actitud crítica entre los alumnos. Sin embargo, en algunos grupos destaca inicialmente una actitud corporativista que con el tiempo tiende a reducirse.

Los alumnos son más críticos con su contribución al trabajo dentro del grupo (autoevaluación) que con respecto al resto de compañeros (co-evaluación).

Los alumnos que sacan mejores notas en los trabajos académicos son más exigentes evaluando a sus compañeros y a ellos mismos, y viceversa, salvo alguna excepción.

Los resultados muestran que un grupo que inicialmente trabaja bien en equipo, con el tiempo, refuerza más esta competencia tendiendo hacia la evaluación Excelente, y viceversa.

Los resultados muestran que las co-evaluaciones, autoevaluaciones y 'rúbricas de evaluación' permitirán obtener evaluaciones más justas de los trabajos académicos al ser más objetivas por involucrar a ambas partes alumnado y profesorado.

\section{Referencias}

CUARTAS URIBE, B et al. (2014). "¿Se puede evaluar el trabajo en grupo de forma individual? Experiencia piloto en una asignatura experimental" Congreso docente In-Red 2014. Valencia, España. Universitat Politècnica de València. 1114-1120.

MARTÍNEZ FIGUEIRA, M.E., RAPOSO RIVAS, M. (2010). "Seguimiento de trabajos tutelados en grupo mediante rúbricas" Congreso docente La docencia en el nuevo escenario del Espacio Europeo de Educación Superior. Vigo, España. Vicerreitoría de Formación e Innovación Educativa. Universidade de Vigo, 567-570.

MORALES-VALLEJO, P. (2008). "Estrategias para evaluar y calificar el producto del equipo: cómo diferenciar las calificaciones individuales". Universidad Pontificia Comillas.

PÉREZ CANTO, S. et al. (2010). "Análisis del papel que desempeñan las habilidades sociales en el Espacio Europeo de Educación Superior". Congreso docente La docencia en el nuevo escenario del Espacio Europeo de Educación Superior. Vigo, España. Vicerreitoría de Formación e Innovación Educativa. Universidade de Vigo, 53-63.

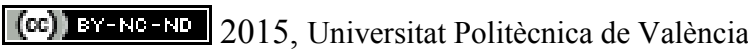


Autoevaluación y co-evaluación continua del trabajo individual y grupal en una asignatura experimental.

ALGUACIL DE NICOLÁS, M. et al. (2010) "Propuestas metodológicas afines al Espacio Europeo de Educación Superior: percepción del alumnado". Congreso docente La docencia en el nuevo escenario del Espacio Europeo de Educación Superior. Vigo, España. Vicerreitoría de Formación e Innovación Educativa. Universidade de Vigo, 191-194.

IEMA. Grupo de Innovación en la evaluación para la mejora del aprendizaje activo. (2006) "Evaluación compartida del trabajo: investigación multidisciplinar". Editorial Universitat Politècnica de València, Valencia. 